\title{
Observations and Interpretation of Subsurface Magnetic Structures
}

\author{
P. S. Cally \\ Centre for Stellar and Planetary Astrophysics, School of Mathematical \\ Sciences, Monash University, Victoria, Australia 3800
}

\author{
A. D. Crouch
}

Department de Physique, Université de Montreal, Canada

\begin{abstract}
Immediate subsurface local solar structures - flow, magnetic, and thermal - especially those associated with active regions, are of considerable interest, and are potentially accessible using high $\ell$ helioseismology. In recent years, classical modal seismology has been supplemented with new approaches, such as time-distance helioseismology, Hankel analysis, and acoustic holography. Results from time-distance helioseismology on shallow subsurface wave speed are at odds with those from the other methods. A simple model is presented which yields good agreement with the observed Hankel phase shifts of $p$ modes passing through sunspots.
\end{abstract}

\section{Introduction}

The subsurface structure of solar active regions has been the subject of conjecture for decades. But now, the advent of various local helioseismic techniques has opened up the possibility of probing sunspots and their surrounds using observations of surface velocities. Three specific techniques - Time-Distance Helioseismology (TD), Acoustic Holography (AH), and Hankel Analysis - have made particular contributions. The foundations of these three approaches are quite different, and taking information from each can give us a more detailed picture than can be gleaned from any one technique alone. Importantly, they also serve as checks on each other, and the purpose of this paper is, in part, to point out a discrepancy between TD on the one hand and AH and Hankel analysis on the other concerning wave propagation speeds in the immediate subsurface layers of sunspots.

Perhaps the best developed active region seismology program is based on TD methods (Kosovichev, Duvall \& Scherrer 2000; Kosovichev 2002; and Zhao \& Kosovichev 2003). Time-distance helioseismology in its basic form inverts surface cross-covariance data using a ray-theoretic description of the propagation of disturbances in the solar interior. Variations of travel time are used to infer the positions and magnitudes of regions of enhanced propagation speed, and comparisons of travel times in opposite directions reveal details of flow patterns. TD has for example mapped inflows surrounding spots at the surface, and outflows several $\mathrm{Mm}$ down, forming a cell pattern which is undoubtedly implicated in keeping the spot together over time-scales of weeks. The other major result 
from these TD studies is the inference of a wave speed deficit in the first $\sim 4$ $\mathrm{Mm}$ beneath a spot, and an excess from there down to around $10 \mathrm{Mm}$.

However, both Hankel analysis (Braun 1995) and holography (Braun \& Lindsey 2000a,b) clearly infer wave speed increases at shallow depth, as well as substantial wave absorption. In this paper, we focus on the best available Hankel analysis data, in concert with recent theoretical results from Crouch \& Cally (2003), in an attempt to build a simple model which explains these features. Finally, we speculate on the reasons for the discrepancy with TD.

\section{Hankel Data}

Hankel analysis (Braun, Duvall \& LaBonte 1987; Braun et al. 1992; and Braun 1995 ) is the only one of the three techniques which is based on global $p$ modes. It is also the only one which has not yet been married with an inversion method to directly image the subsurface layers. Nevertheless, when regarded as a forward technique, it can certainly provide useful information, and potentially rule out many models. The major result from Hankel analysis of sunspots is that they both partially absorb $p$ modes incident upon them, and shift their phases forward on emergence.

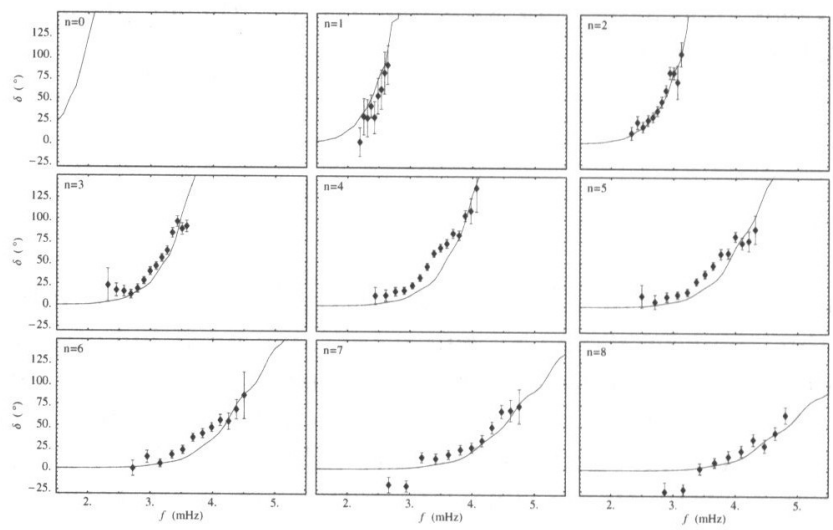

Figure 1. Hankel phase shift $\delta$ (degrees) as a function of frequency for radial orders 0 to 8 ( $n=0$ refers to the $f$-mode). The points with error bars are for the active region NOAA5254 and are taken from Braun (1995) (the errors on the $f$-mode frequency shifts are too large for that data to be useful). The curves are from the model of $\S 3$

There are several effects which disguise the true absorption, for example the presence of acoustic glories around spots which produce enhanced emission at some frequencies. For this reason, we regard the phase shift as a more direct measure of the influence of a sunspot. In Fig. 1 phase shift $\delta$ is plotted as a function of frequency $f$ for one of the active regions discussed in Braun (1995), with each panel referring to a different radial order $n$ of $p$-mode. Two features are particularly instructive: (i) for each $n$, the phase shift increases with frequency; (ii) this rising curve shifts rightward with increasing $n$. The interpretation is clear. For fixed $n$, increasing frequency corresponds to increasing spherical har- 
monic degree $\ell$ and to decreasing lower turning point depth. Hence, the higher frequency modes are trapped ever closer to the surface. Consequently, we interpret the rise in $\delta$ with $f$ as indicating an increased wave travel speed compared to the quiet sun immediately below the active region. For higher radial order $n$, the $p$ modes extend deeper for fixed $f$, and so it is necessary to increase $f$ further in order to bring the mode up into the enhanced speed region. This explains the rightward shift with $n$. However, our interpretation appears to be at odds with the TD result.

\section{Simple Model}

The Sun's $p$ modes are substantially altered in the presence of strong magnetic field. For example, if we superpose a uniform vertical magnetic field on a simple polytropic model (Cally, Bogdan \& Zweibel 1994), the horizontal wavenumber $k$ of the modes for fixed frequency typically reduces, resulting in an increased horizontal phase speed. In addition, $k$ acquires an imaginary part, indicating decay with horizontal distance $x$. This is due to coupling to the magnetoacoustic slow wave, which siphons off much of the $p$-mode's energy and directs it downwards along the magnetic field lines into the interior. This mechanism is capable of explaining the observed absorption of $f$ modes in sunspots, but is too weak for the $p$ modes, especially beyond the first one or two radial orders.

Recently, Crouch \& Cally (2003) have recalculated the eigenvalue wavenumbers for inclined uniform magnetic field. It is found that absorption is greatly enhanced at moderate inclination angles, with the peak effect being at around $30^{\circ}$ from the vertical, depending on frequency and radial order. As shown in Cally et al. (2003), such inclined field produces ample absorption to explain the observations (indeed, too much at frequencies beyond a few $\mathrm{mHz}$, though this can be explained by reference to acoustic glories and the other mechanisms which serve to mask the true extent of absorption).

Using the inclined-field eigenvalue wavenumbers, Cally et al. (2003) developed a simple model which postulates that these eigenvalues can be used locally as a crude measure of both phase shift (through the real part of $k$ ) and absorption (imaginary part). In so doing, many complications are ignored, but this model is built as an attempt to understand the essence of the physics involved, and in particular to determine the simplest description which can give at least qualitatively correct results. Work is currently underway to improve on some of these approximations, most notably by adopting a more realistic model than the polytrope used in the eigenvalue calculations.

The upshot of the model is that, for given "sunspot" radius $R$, magnetic field inclination $\theta$, and field strength parameter $L$ (this is the depth in the polytrope at which the sound and Alfvén speeds coincide), plots of phase shift $\delta$ and absorption coefficient $\alpha$ against frequency can be produced for each radial order $n$. To determine $R$ it is best to first compare with the $m$-specific data. Here $m$ is the cylindrical order (through $\exp [\operatorname{im} \theta]$ ) of the modes. Direct (axisymmetric) incidence of $p$ modes on a circular spot corresponds to $m=0$, with higher $m$ being associated with increasingly glancing incidence. The data indicates that $\delta$ has a steep shoulder at $m$ of order 10 , beyond which there is effectively no shift. Detailed fits of our model to this data indicate that $R$ should be chosen to 
be around 25 to $30 \mathrm{Mm}$ for the active region NOAA5254. This is substantially larger than the observed penumbral radius of $18 \mathrm{Mm}$, but is in accord with other estimates of the $p$-mode scattering radius.

Having settled on $R=27.5 \mathrm{Mm}, \theta$ and $L$ are adjusted to give the best fits to the observational phase shift data (binned over small $\mathrm{m}$ ). An example of such a fit is given in Fig. 1 and corresponds to $L=0.58 \mathrm{Mm}$ and $\theta=35^{\circ}$. The agreement is remarkable. This encourages us to believe that the essential physics of the interaction between $p$ modes and sunspots is being captured in this model, despite its simplicity. However, the precise values of the optimal $L$ and $\theta$ are likely to be revised when more realistic solar models are used to calculate the eigenvalues. We also point out that Cally, Crouch \& Braun (2003) present a "shell" model in which concentric shells in the sunspot are given different $L$ and $\theta$, which allows more detailed fine tuning. We defer extensive treatment of this model till the more realistic eigenvalue tables are available.

\section{Discussion}

The sunspot model, despite its simplicity, clearly gives excellent agreement with the Hankel phase shift data, and also provides ample absorption. However, at this stage it cannot be used to quantitatively probe sunspot interiors, largely because it is based on a polytropic models rather than something more realistic.

Both the Hankel and Acoustic Holography approaches are based on viewing a sunspot from the outside. On the other hand, it would appear that TD results for wave travel speeds in the shallow layers of a spot are based on ray paths which begin and end in the spot. Since the TD raypath formalism does not as yet adequately address magnetic field, we are concerned that this may result in errors. In addition, it is not clear how substantial fast-to-slow mode conversion in these layers will affect TD inversions. Perhaps these are the reasons for the wave speed discrepancy between TD and the other methods.

\section{References}

Braun, D. C. 1995, ApJ, 451, 859

Braun, D. C., Duvall, T. L. \& LaBonte, B. J. 1987, ApJ, 319, L27

Braun, D. C., Duvall, T. L., LaBonte, B. J., Jefferies, S. M., Harvey, J. W. \& Pomerantz, M. A. 1992, ApJ, 391, L113

Braun, D. C. \& Lindsey, C. 2000a, Solar Phys., 192, 285

Braun, D. C. \& Lindsey, C. 2000b, Solar Phys., 192, 307

Cally, P. S., Bogdan, T. J. \& Zweibel, E. G. 1994, ApJ, 437, 505

Cally, P. S., Crouch, A. D. \& Braun, D. C. 2003, MNRAS, in press

Crouch, A. D. \& Cally, P. S. 2003, Solar Phys., 214, 201

Kosovichev, A. G. 2002, Astron. Nachr., 323, 186

Kosovichev, A. G., Duvall, T. L. \& Scherrer, P. H. 2000, Solar Phys., 192, 159

Zhao, J. \& Kosovichev, A. G. 2003, ApJ, 591, 446 